\title{
Contrast-enhanced ultrasonography with Sonazoid in hepatocellular carcinoma diagnosis
}

\author{
Yasunori Minami, Masatoshi Kudo \\ Department of Gastroenterology and Hepatology, Kindai University Faculty of Medicine, Osaka 589-8511, Japan.
}

Correspondence to: Prof. Yasunori Minami, Department of Gastroenterology and Hepatology, Kindai University Faculty of Medicine, 377-2 Ohno-higashi Osaka-sayama, Osaka 589-8511, Japan. E-mail:minkun@med.kindai.ac.jp

How to cite this article: Minami Y, Kudo M. Contrast-enhanced ultrasonography with sonazoid in hepatocellular carcinoma diagnosis. Hepatoma Res 2020;6:46. http://dx.doi.org/10.20517/2394-5079.2020.32

Received: 25 Mar 2020 First Decision: 11 May 2020 Revised: 12 May 2020 Accepted: 23 Jun 2020 Published: 7 Aug 2020

Academic Editor: Yuko Kono Copy Editor: Cai-Hong Wang Production Editor: Tian Zhang

\begin{abstract}
With the development of second-generation contrast agents and advancement in contrast harmonic imaging, contrast-enhanced ultrasonography (CEUS) now has the capacity to sensitively and accurately show tumor vascularity. Therefore, marked improvements have been achieved in the diagnosis of focal liver lesions(FLLs), including hepatocellular carcinoma (HCC), by US. In contrast to other agents, Kupffer cells in liver sinusoids take up Sonazoid. Two contrast enhancement phases occur in CEUS with Sonazoid: a vascular phase and Kupffer phase. Images obtained in the Kupffer phase have higher diagnostic sensitivity for hepatic malignancies because the majority of these malignancies do not contain Kupffer cells. Dynamic images obtained in the vascular phase markedly narrow the clinical differential diagnoses of FLLs. The sustainable detection of inconspicuous HCC, adequate guidance of ablation therapy, and accurate assessment of treatment responses in HCC are all facilitated by Sonazoid. The principles, clinical applications, and techniques of CEUS with Sonazoid in the diagnosis of HCC will be reviewed herein.
\end{abstract}

Keywords: Contrast-enhanced ultrasonography, focal liver lesion, hepatocellular carcinoma, sonazoid

\section{INTRODUCTION}

Recent advances in the multi-modality treatment of hepatocellular carcinoma (HCC) have contributed to significant improvement in the prognosis of patients with this type of primary liver cancer. The importance of the early detection of liver nodules, accurate diagnosis of HCC, and tumor staging for treatment planning is increasingly recognized. Guidelines for the utilization of imaging tests in the diagnosis of HCC have been developed by a number of societies, including the American Association for the Study of Liver Diseases (AASLD), the European Association for the Study of the Liver, the Asian-Pacific Association for the Study of Liver, and the Japan Society of Hepatology ${ }^{[1-4]}$. Due to advances in techniques that have contributed to the

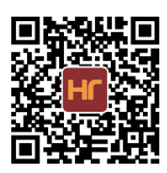


highly sensitive and specific diagnosis of HCC, clinical HCC guidelines now recommend imaging tests. The specificity of single contrast-enhanced imaging is adequate for diagnosing HCC when typical features are observed on dynamic images. Recently, a critical milestone was achieved with integration of Liver Imaging Reporting and Data System (LI-RADS) into the AASLD HCC clinical practice guideline. LI-RADS is a comprehensive algorithm for standardizing the terminology, technique, interpretation and reporting for patients at high risk for $\mathrm{HCC}^{[5]}$.

Contrast-enhanced ultrasonography (CEUS) is now widely used in clinical practice and has markedly expanded the scope of the diagnosis of focal liver lesions(FLLs) by US. In CEUS, second-generation contrast agents, including SonoVue (sulfur hexafluoride), Definity (perflutren lipid), and Sonazoid (perflubutane), are microbubbles composed of a low-solubility gas enveloped by a phospholipid shell ${ }^{[6]}$. Kupffer cells in the reticuloendothelial system of the liver take up Sonazoid, which remains in these cells for several hours, resulting in two contrast enhancement phases: a vascular phase and Kupffer phase. Sonazoid is advantageous for the diagnosis of HCC because of the higher diagnostic sensitivity of images obtained in the Kupffer phase for hepatic malignancies; the majority of hepatic neoplasms, particularly malignant tumors, do not contain Kupffer cells ${ }^{[7]}$. CEUS LI-RADS is a standardized system for CEUS exams and can allow for accurate categorization of observations in patients with chronic hepatitis B or cirrhosis ${ }^{[8]}$. The utility of SonoVue and Definity is supported by CEUS LI-RADS, whereas Sonazoid alone has not been included yet. It is expected that Sonazoid utilization will be incorporated in the next version of CEUS LI-RADS.

Sonazoid was approved for use as an ultrasound contrast agent in Japan in $2007^{[9]}$, followed by Norway, Korea, and Singapore. It was subsequently approved in Taiwan in 2018 and China in 2019. CEUS with Sonazoid is now regarded as a valuable diagnostic tool in the management of HCC patients. The principles, clinical applications and techniques of CEUS with Sonazoid in the management of HCC will be reviewed herein.

\section{STRUCTURE AND PHARMACOKINETICS OF SONAZOID}

Sonazoid (GE Healthcare, Waukesha, WI, USA) consists of lipid-coated microbubbles containing perfluorocarbon within a well-defined size range (median diameter of approximately $3 \mu \mathrm{m}$ ). These microbubbles are stabilized by a monomolecular membrane of hydrogenated egg phosphatidyl serine that is embedded in an amorphous sucrose structure ${ }^{[6]}$. Sonazoid powder is reconstituted with $2 \mathrm{~mL}$ of sterile water for administration by injection. The clinical dose of Sonazoid that is generally employed to image liver lesions is $0.015 \mathrm{~mL} / \mathrm{kg}$ body weight; however, the recommended dose is decreased to $0.0075-0.0010 \mathrm{~mL} / \mathrm{kg}$ body weight when an US machine with high sensitivity for the detection of contrast agents is used.

Regarding the two contrast enhancement phases in real-time CEUS, the vascular phase (between $10 \mathrm{~s}$ and 5-7 min after the injection of Sonazoid) shows tumor vascularity, while the Kupffer phase (from 10 min after the injection) shows hepatic parenchymal findings because Kupffer cells or liver sinusoids take up this contrast agent. The artery- and portal-dominant time zones in the vascular phase are referred to as the arterial and portal phases, respectively [Figure 1] ${ }^{[10,11]}$. Contrast enhancement in the Kupffer phase provides important information on FLLs because hypoenhancement indicates HCC, while benign lesions mostly show iso- or hyperenhancement. Imaging patterns, namely, arterial enhancement with Kupffer defects, are an important distinguishing feature of hepatic malignancies.

Dynamic CEUS displays similar, but distinct, vascular patterns to contrast-enhanced computed tomography (CECT); the contrast agents used in US are retained within blood vessels (blood pool contrast agents), whereas those for CT and magnetic resonance imaging (MRI) move into the extracellular space until their concentrations balance between the intravascular and extracellular spaces ${ }^{[12]}$. 


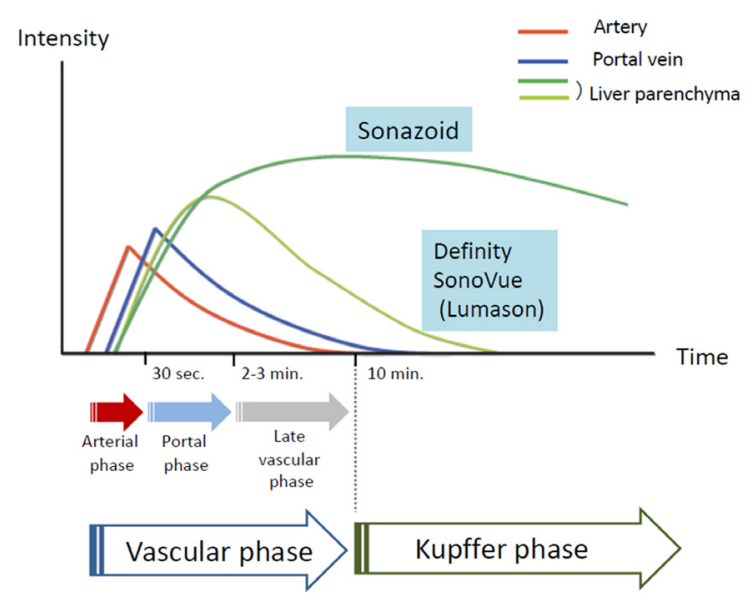

Figure 1. Pharmacokinetic behaviors of US contrast agents. Vascular and Kupffer phase images may be obtained using Sonazoid, but not Difinity/SonoVue (Lumason). Sonazoid microbubbles are taken up by Kupffer cells and show homogeneous enhancement in a normally functioning liver parenchyma. Kupffer phase images are generally obtained $10 \mathrm{~min}$ after the injection of Sonazoid, the stability of which does not degrade for at least $60 \mathrm{~min}$

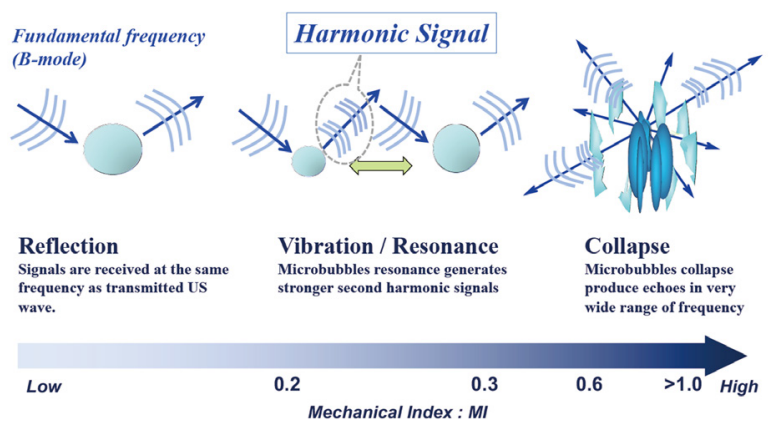

Figure 2. Behavior of microbubbles exposed to ultrasound. Microbubbles oscillate in a symmetrical manner at very low $\mathrm{MI}(<0.1)$ with stable linear scattering. In contrast, asymmetrical oscillations are observed at low/medium MI (0.2-0.6), with microbubbles expanding more than they contract because they are more resistant to compression. This asymmetry causes harmonic emissions. Transient nonlinear scattering occurs at high $\mathrm{MI}(>0.6)$, and is followed by microbubble destruction. MI: mechanical index

\section{MEDICAL ULTRASOUND TECHNOLOGIES}

The mechanical index (MI) is a measure of the insonation power of microbubbles within an ultrasound field [Figure 2]. Second-generation microbubble contrast agents remain static when MI is very low and scatter the ultrasound beam. As MI increases, microbubbles linearly (MI < approximately 0.2) or nonlinearly (approximately $0.2<\mathrm{MI}<0.5$ ) oscillate at their resonance frequency. Real-time scanning with CEUS generally involves low MI of < 0.3. Microbubbles strongly oscillate at MI of higher than 0.6, expand beyond their limit, and ultimately burst. CEUS images may be generated from signals of the non-linear oscillation of microbubbles or their destruction ${ }^{[12]}$.

Information obtained on harmonic distortions in echo signals is used in non-linear ultrasonic imaging techniques. The non-linear mechanical behavior of microbubbles in contrast-enhanced imaging or nonlinear wave propagation within tissues in tissue harmonic imaging causes harmonic distortions in ultrasound signals. Since echo signals contain both linear and non-linear reflections, the extraction of non-linear echo content is important for ensuring the sufficient performance of harmonic imaging. CEUS-specific modes including pulse inversion, amplitude modulation, and pulse inversion amplitude modulation have been devised to suppress linear tissue signals, thereby enhancing the detection of non-linear microbubble echoes $^{[13-15]}$ [Figure 3]. The non-CEUS-specific mode, tissue harmonic imaging (THI), which is a non- 

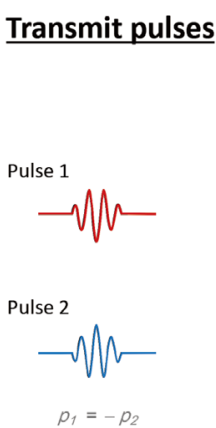

$\underline{\text { Receive pulses }}$

Pulse inversion

$=$ Pulse $1+$ Pulse 2

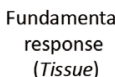

response
(Tissue)

Harmonic

response

(Microbubble)

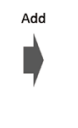

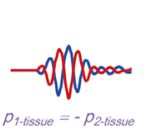

1
B

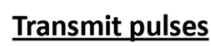

Receive pulses

Amplitude modulation $=[$ Pulse 1] $-2 \times[$ Pulse 2]

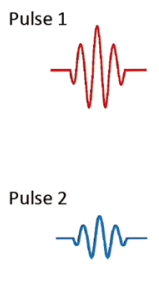

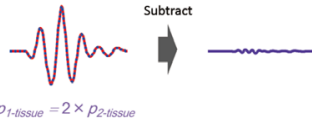

$\rho_{\text {t-tissue }}=2 \times \rho_{2-\text { tissu }}$

Harmonic

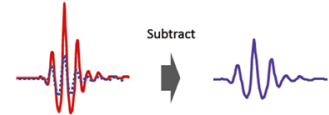

$p_{1-\text { bubble }}>2 \times p_{2-\text { buble }}$

Figure 3. Pulse inversion and amplitude modulation. A: pulse-inversion technique is used in second harmonic imaging. Pulse 1 excites microbubbles, generating a linear fundamental response along with higher harmonic components. The inverted pulse 2 generates the same frequency components, however with different phases. The linear fundamental response from tissue experiences a $180^{\circ}$ phase shift relative to the pulse 1 components, whereas the second harmonic response from microbubble experiences a $360^{\circ}\left(=0^{\circ}\right)$ phase shift. As a result, the fundamental responses are canceled out and the second harmonic responses are constructively added together; B: an amplitude modulation technique also plays a role in ultrasonic nonlinear imaging. An amplitude pulse is transmitted to eliminate the linear response and to elicit a nonlinear response. Upon reception, the pulse 2 components are rescaled and subtracted. Then, the fundamental response from tissue is canceled, and the second harmonic response from microbubble is leaked out
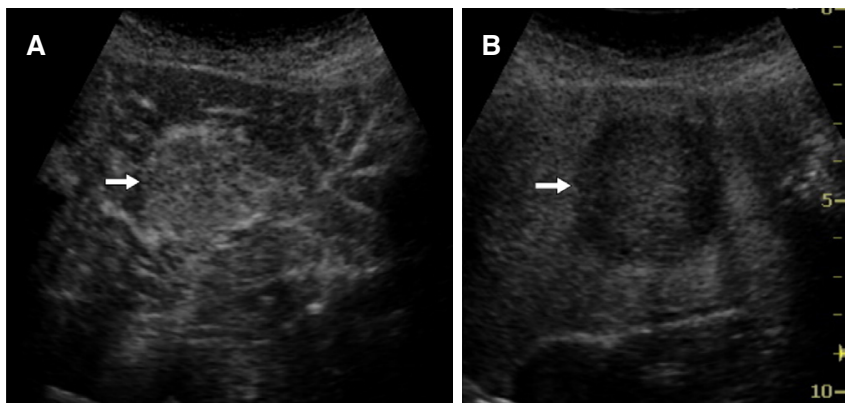

Figure 4. Hepatocellular carcinoma. A: contrast-enhanced ultrasonography shows homogenous strong enhancement (arrow) in the early arterial phase; B: a clear defect (arrow) with an irregular border is observed on a Kupffer phase image

contrast form of native harmonics, is also applied to contrast imaging. THI is advantageous because it provides a better signal-to-noise ratio. Contrast THI with Sonazoid offers a better contrast-to-tissue ratio at the cost of blood flow signals. Therefore, contrast THI provides an overlay view of conventional THI and contrast imaging $^{[16]}$. It allows us to observe vessels and inconspicuous lesions in the liver through all phases at high spatial and time resolutions; however, image contrast in the Kupffer phase may be slightly lower than that of the pulse inversion ${ }^{[17]}$.

\section{CLINICAL APPLICATION OF CEUS}

\section{Diagnosis of HCC}

Classic HCC generally receive a blood supply from abnormal arteries alone and are diagnosed based on positive enhancement (hypervascularity) in the arterial phase and defects in the Kupffer phase [Figure 4]. Previous studies reported the accurate diagnosis of small HCC $(\leq 2 \mathrm{~cm})$ using CEUS at a sensitivity of $81 \%-95 \%$ and specificity of $82 \%-86 \%{ }^{[18,19]}$. However, a potential limitation of this imaging modality is that a small subset of atypical HCC does not show hypervascularity in the arterial phase. Tumor hemodynamics change through the process of multistep hepatocarcinogenesis from low grade dysplastic nodule (DN) to moderately differentiated HCC [Figure 5]. The enhancement patterns of HCC are influenced by the degree of cellular differentiation. Arterial and portal blood supplies in pathological early or well-differentiated HCC vary, which increases the challenges associated with reaching an accurate diagnosis. The nodule-in- 


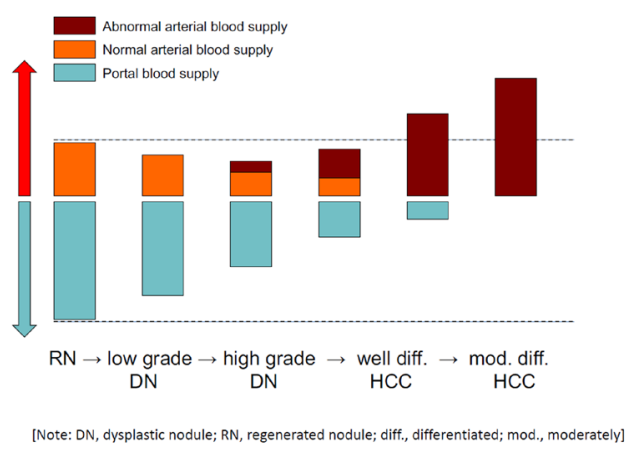

Figure 5. Tumor blood flows and multistep hepatocarcinogenesis. The development of hepatocellular carcinoma (HCC) occurs through a multistep process in the following sequence: large regenerative nodule (RN), low- or high-grade dysplastic nodule (DN), DN with a focus of HCC, well differentiated HCC, and moderately to poorly differentiated HCC. During the dedifferentiation, the intratumoral areas supplied portal blood flow are gradually reduced, whereas the intratumoral areas supplied arterial blood flow are synchronously changed. Normal arterial blood supply is reduced at an early stage, and then abnormal arterial blood supply is finally replaced
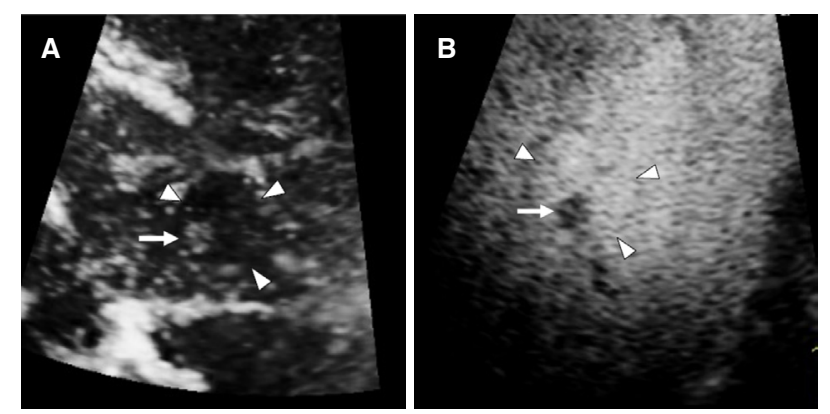

Figure 6. Nodule-in-nodule appearance of hepatocellular carcinoma. A: Arterial phase image shows an inner hypervascular spot (arrow) within the outer hypovascular nodule (arrowheads); B: Kupffer phase image shows better differentiation between a small defect (arrow) and the outer hypointense nodule (arrowheads)

nodule hemodynamic pattern, which is characterized by hyperintense foci in hypointense nodules, is specific for diagnosing early-stage HCC $^{[20]}$ [Figure 6]. Arterial enhancement is less commonly observed for welldifferentiated HCC; nodules are more likely to be isoenhanced or slightly hypoenhanced from the portal to Kupffer phases ${ }^{[21,22]}$. Malignant liver lesions including HCC, cholangiocarcinoma, metastasis, etc. appear as hypoechoic areas surrounded by hyperechoic background liver in the Kupffer phase due to the depletion of Kupffer cells within them ${ }^{[11,12]}$. The Kupffer defect can be easily detected and increasing its diagnostic performance. Moreover, the images of Kupffer defect are also useful to evaluate the macroscopic type of HCC that is a significant prognostic factor of HCC patients ${ }^{[23]}$.

Repeated contrast injections may be performed when an enhancement defect is identified in the Kupffer phase. This procedure is termed "defect reperfusion imaging" or "the re-injection technique", and arterial enhancement may be superimposed on Kupffer images of lesions ${ }^{[24,25]}$. Defect reperfusion imaging generates a very high detection rate of HCC that is not achievable with conventional B-mode US ${ }^{[26]}$ [Figure 7]. Kupffer phase image surveillance is also useful for the early detection and confirmation of HCC with the reinjection technique ${ }^{[27]}$.

\section{Characterization of FLLs}

The accurate differential diagnosis of FLLs requires clinical imaging tests, and CEUS plays an important role in the characterization of FLLs. CEUS with Sonazoid can show a high sensitivity of the detection of intranodular blood flow. Most malignant liver lesions are demonstrated as hypoechoic masses from the portal phase to the Kupffer phase, while most benign liver lesions are iso- or hyperechoic during these phases. 

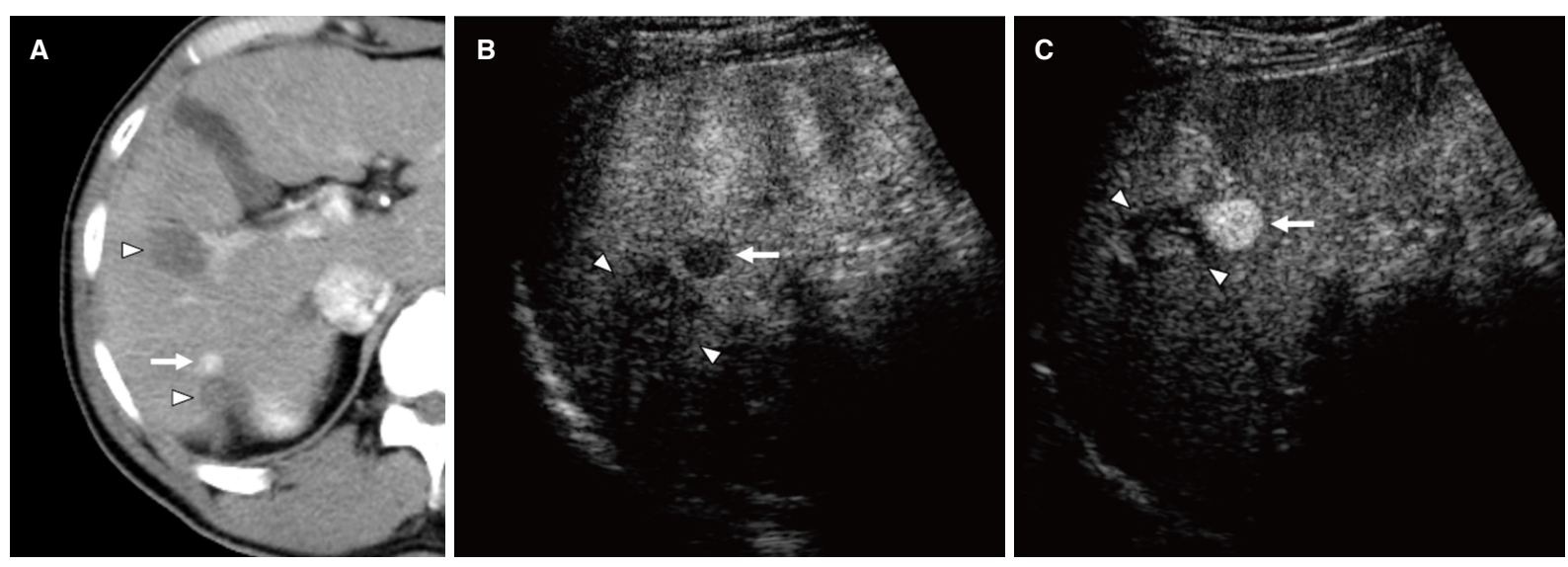

Figure 7. Defect reperfusion imaging for the local progression of hepatocellular carcinoma (HCC) after radiofrequency ablation (RFA). A: a computed tomography image shows focal arterial enhancement of the local progression of HCC (arrow) and RFA-induced coagulation necrosis (arrow heads); B: viable HCC (arrow) in close proximity to the necrotic area (arrow heads) are shown as areas with defects in the Kupffer phase; C: recurrent HCC (arrow) is clearly identified using the Sonazoid reinjection technique, whereas the necrotic area (arrow heads) does not enhance
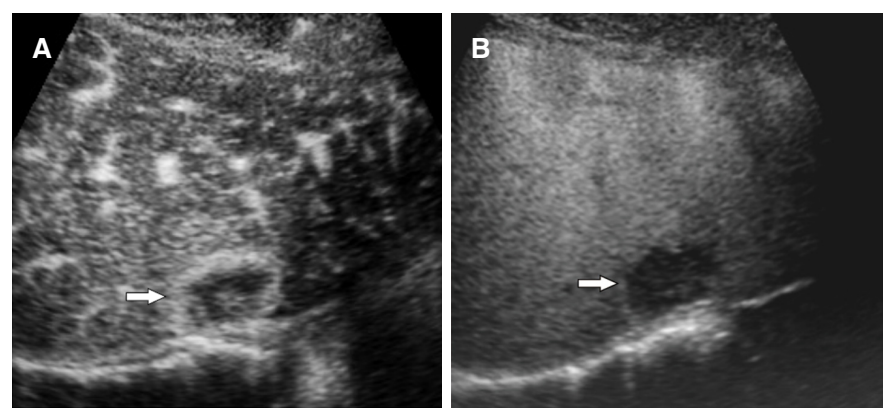

Figure 8. Liver metastasis from colon cancer. A: liver metastasis shows peripheral irregular rim-like enhancement (arrow); B: a clear defect (arrow) is evident in the Kupffer phase

Arterial hypervascularity is generally observed in the tumor periphery of adenocarcinoma liver metastases from many organs including colon, stomach, pancreas, etc., at which tumor cells are abundant, while complete defects are detected in the Kupffer phase ${ }^{[11]}$ [Figure 8]. Washout is typically more rapid for liver metastases than for HCC, for which it is often slow ${ }^{[21,28]}$. However, homogeneous hypervascularity in the arterial phase was previously reported in between $10 \%$ and $15 \%$ of adenocarcinoma liver metastases ${ }^{[22]}$. In addition, renal cell carcinoma or gastrointestinal stromal tumor typically cause hypervascular metastases, and these lesions tend to show homogeneous arterial enhancement that is similar to typical HCC.

Similarities have been identified in the enhancement patterns of intrahepatic cholangiocarcinoma and metastatic liver cancer on CEUS, with rim-like enhancement in the early arterial phase and complete defects in the Kupffer phase commonly being detected ${ }^{[11]}$. Nevertheless, it is important to note that approximately $30 \%$ of intrahepatic cholangiocarcinomas show hypervascularity and enhancement in the arterial phase ${ }^{[22]}$, consistent with the typical enhancement pattern of HCC on CEUS. Rapid washout has also been reported for intrahepatic cholangiocarcinoma ${ }^{[29]}$. Peripheral rim-like enhancement and quick contrast washout may provide high efficiency in the differentiation of intrahepatic cholangiocarcinoma from HCC.

Hepatocellular adenoma (HCA) often shows arterial hyperenhancement ${ }^{[28]}$ and approximately $30 \%$ of HCAs show mild washout in the late vascular phase of $\mathrm{CEUS}^{[28]}$, making it difficult to distinguish HCA from welldifferentiated HCC. In such causes, the individual patient's background such as a history of risk factors for $\mathrm{HCC}$ is very important for differentiation, and a biopsy may be necessary for the precise diagnosis. 

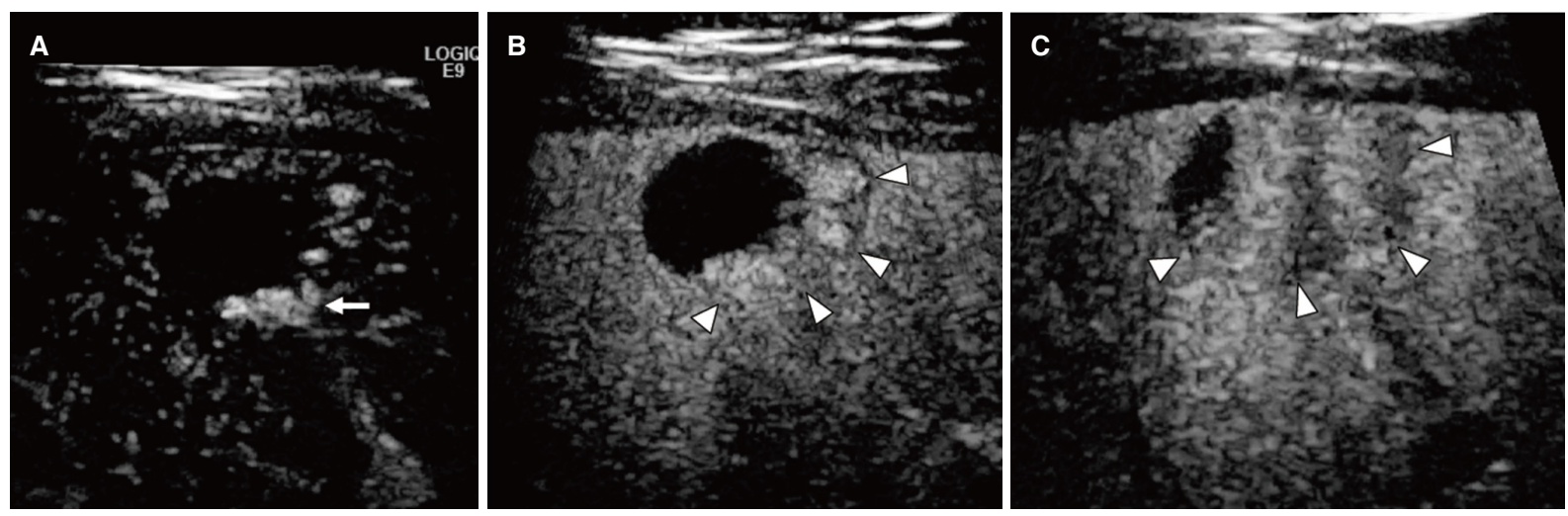

Figure 9. Hepatic hemangioma. A: early arterial phase image shows the typical "peripheral globular enhancement" (arrow) of the lesion; B: the lesion shows "partial centripetal filling" (arrow heads) during the portal phase; C: a progressive filling pattern (arrow heads) is observed in the Kupffer phase
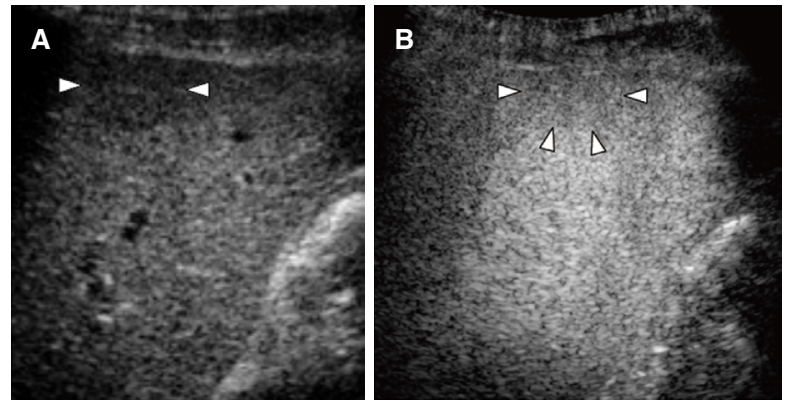

Figure 10. Dysplastic nodule. A: dysplastic nodule (arrowheads) shows hypovascularity in the arterial phase because of the reduction of arterial and portal supplies; B: the Kupffer phase image shows a slightly hypointense lesion with unclear border (arrowheads)

Liver hemangioma shows peripheral nodular enhancement in the arterial phase and centripetal filling through the portal to Kupffer phases of CEUS [Figure 9] ${ }^{[11]}$. Although incomplete late filling has been reported in some cases of larger hemangioma, the enhancement pattern of "peripheral nodular arterial enhancement" in combination with "complete filling" resulted in a sensitivity of $98 \%$ for the diagnosis of liver hemangioma ${ }^{[30]}$.

The typical findings of focal nodular hyperplasia on CEUS include hypervascularity in the early arterial phase with a spoke-wheel pattern and an iso-enhanced mass with a hypo-enhanced central scar through the portal to Kupffer phases ${ }^{[11]}$. The sensitivity of CEUS for detecting the spoke-wheel pattern is markedly higher than that of color-Doppler imaging.

Hepatic angiomyolipoma (AML) is a relatively rare benign mesenchymal tumor of the liver. Pathologically, it is composed of varying proportions of fat, muscle, and blood vessels. AML is characterized by a hyperechoic nodule on B-mode US due to a fatty component and is generally observed as a hypervascular lesion in the arterial phase and a defective lesion in the Kupffer phase ${ }^{[4]}$. However, the diagnosis of hepatic AML is still challenging because imaging characteristics can vary depending on the proportions of its components.

Normal hepatic arteries and portal veins are generally present within low-grade DNs which do not show the early uptake of contrast agents in the arterial phase. A previous study indicated that a high-grade DN showed transient hypovascularity in the arterial phase, and this finding was attributed to increased cellularity ${ }^{[11]}$ [Figure 10]. 
Inflammatory pseudotumor (IPT) of the liver is a rare benign nodule and can display various enhancement patterns on CEUS due to pathological change during the course of disease progression. When the nodules are abundant in inflammatory cells and granulation tissues, they often appear as an area of diffuse homogeneous hyperenhancement. As more necrosis and fibrosis develop within the nodules, IPT may show heterogeneous or peripheral rim-like enhancement ${ }^{[31]}$.

Meta-analytic studies reported the ability of CEUS to accurately differentiate between benign and malignant FLLs at a sensitivity of $93 \%$ and specificity of $90 \%$, and also demonstrated its similar diagnostic performance to dynamic CECT and MRI ${ }^{[32,33]}$. Furthermore, the diagnostic accuracy of CEUS for lesions that were inconclusive on CECT increased from $42 \%-44 \%$ to $89 \%-92 \%$, and a higher diagnostic confidence level was confirmed $^{[34,35]}$.

\section{CEUS guidance of biopsy/ablation therapy}

The correct placement of the needle into the target tumor for percutaneous biopsy/ablation therapy increases its technical success rate. B-mode US does not accurately detect HCC in the presence of local tumor progression after treatment or true HCC surrounded by large regenerative nodules in cirrhotic livers. The rates of HCC with poor conspicuity on planning B-mode US for ablation therapy ranged from 5.2 to 38.8 in previous reports ${ }^{[36-39]}$. CEUS with Sonazoid facilitates needle placement in HCC that is poorly depicted on B-mode US because the defect lesion functions as a target for insertion.

If imaging studies fail to reveal an accurate diagnosis of FLLs, biopsy may be required. The limitations of percutaneous liver biopsy guided with B-mode US incldue its high rates of false-negative results. However, correct targeting and guiding steps benefit from the use of CEUS with Sonazoid ${ }^{[40,41]}$. The diagnosis of benign FLLs may be improved with the utility of CEUS during liver biopsy without surgical intervention.

A previous study reported that the technical success rate of a single radiofrequency ablation (RFA) session was significantly higher with CEUS than with B-mode US $(94.7 \% \text { vs. } 65.0 \%, P=0.043)^{[42]}$. Furthermore, the number of RFA sessions conducted in a historical cohort was smaller with Sonazoid CEUS guidance than with B-mode US guidance ${ }^{[43,44]}$. Another study showed that the sustained local control rate was markedly higher for CEUS-guided RFA than for B-mode US-guided RFA ( $85.3 \%$ vs. $66.4 \%$ at 2 years $)^{[45]}$. In addition, inconspicuity on B-mode US and CEUS represents one of the most difficult conditions for percutaneous RFA. The combination of fusion imaging and CEUS is an effective guidance in ablation therapy for poorly defined HCCs on B-mode US and CEUS/fusion imaging ${ }^{[46]}$.

CEUS may also help to identify complications immediately after ablation therapy such as active bleeding or segmental infarction of the liver ${ }^{[47]}$. Active hemorrhage should be visualized on CEUS as extravasation of microbubbles and infarcted areas can show no enhancement.

\section{Assessment of HCC treatment responses}

The complete lack of enhancement in all phases on CEUS was previously demonstrated in patients with complete treatment responses following arterial chemoembolization for HCC; while intratumoral residual or nodular peripheral enhancement was detected in patients with residual or recurrent HCC. CEUS allows for the reliable prediction of the risk of recurrence in patients with HCC within a short period of time (approximately 1 week) after TACE ${ }^{[48,49]}$.

Important issues to consider in treatment response assessments of RFA are evaluations of the absence of the vascular enhancement of HCC and the ablative margin. Residual HCC shows a focal defect in the Kupffer phase, representing hypervascular enhancement, with reinjections of Sonazoid. However, consistent and accurate assessments of the ablative margin by CEUS is not always be possible because the tumor boundary may not be clearly identified on US after RFA ${ }^{[50]}$. 
Sorafenib (Bayer, Leverkusen, Germany) is the first oral multikinase inhibitor developed for advanced unresectable HCC. Analyses of time intensity curves and arrival time parametric imaging facilitated assessments of the early responses of advanced HCC to sorafenib ${ }^{[51,52]}$. However, not all lesions of multiple HCC treated with sorafenib exhibit similar behaviors on CEUS; therefore, CEUS may only investigate a few regions of interest and may be limited to treatment response assessments of multiple HCC to systemic chemotherapies.

\section{CONCLUSION}

US is a widely used imaging modality for liver diseases because it is minimally invasive, allows for realtime observations, and provides high-resolution images. However, the accuracy of CEUS may be negatively affected by a number of factors, such as acoustic attenuation, various artifacts, and blind areas. Therefore, the favorable and unfavorable characteristics of CEUS need to be carefully considered. This review comprehensively demonstrates the importance of CEUS with Sonazoid for managing patients with HCC. CEUS provides real-time and high-quality images of FLLs, including HCC, in all phases of enhancement, and has markedly increased the accuracy of US-based detection and characterization. CEUS has the ability to differentiate between benign and malignant liver nodules with high accuracy, which is crucial in the management of these patients. Furthermore, CEUS with Sonazoid provides guidance during therapeutic procedures and facilitates assessments of treatment responses. The adequate guidance of ablation and precise monitoring of treatment responses using CEUS with Sonazoid will contribute to further improvement in the prognosis of patients with HCC.

\section{DECLARATIONS}

\section{Authors' contributions}

Study concepts/study design, data acquisition and manuscript drafting: Minami Y

Manuscript editing: Kudo M

\section{Availability of data and materials}

Not applicable.

\section{Financial support and sponsorship}

None.

\section{Conflicts of interest}

Both authors declared that there are no conflicts of interest.

\section{Ethical approval and consent to participate}

Not applicable.

\section{Consent for publication}

Not applicable.

\section{Copyright}

(c) The Author(s) 2020.

\section{REFERENCES}

1. Bruix J, Sherman M. Management of hepatocellular carcinoma: an update. Hepatology 2011;53:1020-2.

2. European Association For The Study Of The Liver; European Organisation For Research And Treatment Of Cancer. EASL-EORTC clinical practice guidelines: management of hepatocellular carcinoma. J Hepatol 2012;56:908-43.

3. Omata M, Cheng AL, Kokudo N, Kudo M, Lee JM, et al. Asia-Pacific clinical practice guidelines on the management of hepatocellular 
carcinoma: a 2017 update. Hepatol Int 2017;11:317-70.

4. Kudo M, Matsui O, Izumi N, Iijima H, Kadoya M, et al. JSH consensus-based clinical practice guidelines for the management of hepatocellular carcinoma: 2014 update by the liver Cancer study Group of Japan. Liver Cancer 2014;3:458-68.

5. Elsayes KM, Hooker JC, Agrons MM, Kielar AZ, Tang A, et al. 2017 version of LI-RADS for CT and MR imaging: an update. Radiographics 2017;37:1994-2017.

6. de Jong N, Emmer M, van Wamel A, Versluis M. Ultrasonic characterization of ultrasound contrast agents. Med Biol Eng Comput 2009;47:861-73.

7. Lee JY, Minami Y, Choi BI, Lee WJ, Chou YH, et al. The AFSUMB consensus statements and recommendations for the clinical practice of contrast-enhanced ultrasound using sonazoid. Ultrasonography 2020;39:191-220.

8. Wilson SR, Lyshchik A, Piscaglia F, Cosgrove D, Jang HJ, et al. CEUS LI-RADS: algorithm, implementation, and key differences from CT/MRI. Abdom Radiol (NY) 2018;43:127-42.

9. Kudo M. Management of hepatocellular carcinoma in Japan as a world-leading model. Liver Cancer 2018;7:134-47.

10. Minami Y, Kudo M. Review of dynamic contrast-enhanced ultrasound guidance in ablation therapy for hepatocellular carcinoma. World J Gastroenterol 2011;17:4952-9.

11. Terminology and Diagnostic Criteria Committee, Japan Society of Ultrasonics in Medicine. Ultrasound diagnostic criteria for hepatic tumors. J Med Ultrason 2014;41:113-23.

12. Chung YE, Kim KW. Contrast-enhanced ultrasonography: advance and current status in abdominal imaging. Ultrasonography 2015;34:318.

13. Eckersley RJ, Chin CT, Burns PN. Optimising phase and amplitude modulation schemes for imaging microbubble contrast agents at low acoustic power. Ultrasound Med Biol 2005;31:213-9.

14. Needles A, Arditi M, Rognin NG, Mehi J, Coulthard T, et al. Nonlinear contrast imaging with an array-based micro-ultrasound system. Ultrasound Med Biol 2010;36:2097-106.

15. Gong P, Song P, Chen S. Improved contrast-enhanced ultrasound imaging with multiplane-wave imaging. IEEE Trans Ultrason Ferroelectr Freq Control 2018;65:178-87.

16. Kono M, Minami Y, Iwanishi M, Minami T, Chishina H, et al. Contrast-enhanced tissue harmonic imaging versus phase inversion harmonic sonographic imaging for the delineation of hepatocellular carcinomas. Oncology 2017;92:29-34.

17. Tanaka H. Current role of ultrasound in the diagnosis of hepatocellular carcinoma. J Med Ultrason (2001) 2020;47:239-55.

18. Niu Y, Huang T, Lian F, Li F. Contrast-enhanced ultrasonography for the diagnosis of small hepatocellular carcinoma: a meta-analysis and meta-regression analysis. Tumour Biol 2013;34:3667-74.

19. Kan M, Hiraoka A, Uehara T, Hidaka S, Ichiryu M, et al. Evaluation of contrast-enhanced ultrasonography using perfluorobutane $(\operatorname{Sonazoid}(\circledR))$ in patients with small hepatocellular carcinoma: comparison with dynamic computed tomography. Oncol Lett 2010;1:485-8.

20. Zheng RQ, Zhou P, Kudo M. Hepatocellular carcinoma with nodule-in-nodule appearance: demonstration by contrast-enhanced coded phase inversion harmonic imaging. Intervirology 2004;47:184-90.

21. Jang HJ, Kim TK, Burns PN, Wilson SR. Enhancement patterns of hepatocellular carcinoma at contrast-enhanced US: comparison with histologic differentiation. Radiology 2007;244:898-906.

22. Jang JY, Kim MY, Jeong SW, Kim TY, Kim SU, et al. Current consensus and guidelines of contrast enhanced ultrasound for the characterization of focal liver lesions. Clin Mol Hepatol 2013;19:1-16.

23. Hatanaka K, Minami Y, Kudo M, Inoue T, Chung H, et al. The gross classification of hepatocellular carcinoma: usefulness of contrastenhanced US. J Clin Ultrasound 2014;42:1-8.

24. Kudo M, Hatanaka K, Maekawa K. Newly developed novel ultrasound technique, defect reperfusion ultrasound imaging, using sonazoid in the management of hepatocellular carcinoma. Oncology 2010;78:40-5.

25. Claudon M, Dietrich CF, Choi BI, Cosgrove DO, Kudo M, et al. World Federation for Ultrasound in Medicine; European Federation of Societies for Ultrasound. Guidelines and good clinical practice recommendations for Contrast Enhanced Ultrasound (CEUS) in the liver - update 2012: A WFUMB-EFSUMB initiative in cooperation with representatives of AFSUMB, AIUM, ASUM, FLAUS and ICUS. Ultrasound Med Biol 2013;39:187-210.

26. Kudo M. Diagnostic imaging of hepatocellular carcinoma: recent progress. Oncology 2011;81:73-85.

27. Kudo M, Ueshima K, Osaki Y, Hirooka M, Imai Y, et al. B-Mode ultrasonography versus contrast-enhanced ultrasonography for surveillance of hepatocellular carcinoma: a prospective multicenter randomized controlled trial. Liver Cancer 2019;8:271-80.

28. Yang HK, Burns PN, Jang HJ, Kono Y, Khalili K, et al. Contrast-enhanced ultrasound approach to the diagnosis of focal liver lesions: the importance of washout. Ultrasonography 2019;38:289-301.

29. Liu GJ, Wang W, Lu MD, Xie XY, Xu HX, et al. Contrast-enhanced ultrasound for the characterization of hepatocellular carcinoma and intrahepatic cholangiocarcinoma. Liver Cancer 2015;4:241-52.

30. Dietrich CF, Mertens JC, Braden B, Schuessler G, Ott M, et al. Contrast-enhanced ultrasound of histologically proven liver hemangiomas. Hepatology 2007;45:1139-45.

31. Kong WT, Wang WP, Cai H, Huang BJ, Ding H, et al. The analysis of enhancement pattern of hepatic inflammatory pseudotumor on contrast-enhanced ultrasound. Abdom Imaging 2014;39:168-74.

32. Friedrich-Rust M, Klopffleisch T, Nierhoff J, Herrmann E, Vermehren J, et al. Contrast-enhanced ultrasound for the differentiation of benign and malignant focal liver lesions: a meta-analysis. Liver Int 2013;33:739-55.

33. Xie L, Guang Y, Ding H, Cai A, Huang Y. Diagnostic value of contrast-enhanced ultrasound, computed tomography and magnetic 
resonance imaging for focal liver lesions: a meta-analysis. Ultrasound Med Biol 2011;37:854-61.

34. Chung YE, Kim KW. Contrast-enhanced ultrasonography: advance and current status in abdominal imaging. Ultrasonography 2015;34:318.

35. Quaia E, De Paoli L, Angileri R, Cabibbo B, Cova MA. Indeterminate solid hepatic lesions identified on non-diagnostic contrastenhanced computed tomography: assessment of the additional diagnostic value of contrast-enhanced ultrasound in the noncirrhotic liver. Eur J Radiol 2014;83:456-62.

36. Minami Y, Kudo M, Kawasaki T, Chung H, Ogawa C, et al. Treatment of hepatocellular carcinoma with percutaneous radiofrequency ablation: usefulness of contrast harmonic sonography for lesions poorly defined with B-mode sonography. AJR Am J Roentgenol 2004;183:153-6.

37. Kim PN, Choi D, Rhim H, Rha SE, Hong HP, et al. Planning ultrasound for percutaneous radiofrequency ablation to treat small $(\leq 3 \mathrm{Cm})$ hepatocellular carcinomas detected on computed tomography or magnetic resonance imaging: a multicenter prospective study to assess factors affecting ultrasound visibility. J Vasc Interv Radiol 2012;23:627-34.

38. Kim AY, Lee MW, Rhim H, Cha DI, Choi D, et al. Pretreatment evaluation with contrast-enhanced ultrasonography for percutaneous radiofrequency ablation of hepatocellular carcinomas with poor conspicuity on conventional ultrasonography. Korean J Radiol 2013;14:754-63.

39. Rajesh S, Mukund A, Arora A, Jain D, Sarin SK. Contrast-enhanced US-guided radiofrequency ablation of hepatocellular carcinoma. J Vasc Interv Radiol 2013;24:1235-40.

40. Eso Y, Takai A, Takeda H, Matsumoto T, Lee M, et al. Sonazoid-enhanced ultrasonography guidance improves the quality of pathological diagnosis in the biopsy of focal hepatic lesions. Eur J Gastroenterol Hepatol 2016;28:1462-7.

41. Park HS, Kim YJ, Yu MH, Jung SI, Jeon HJ. Real-time contrast-enhanced sonographically guided biopsy or radiofrequency ablation of focal liver lesions using perflurobutane microbubbles (sonazoid): value of Kupffer-phase imaging. J Ultrasound Med 2015;34:411-21.

42. Minami Y, Kudo M, Chung H, Kawasaki T, Yagyu Y, et al. Contrast harmonic sonography-guided radiofrequency ablation therapy versus B-mode sonography in hepatocellular carcinoma: prospective randomized controlled trial. AJR Am J Roentgenol 2007;188:489-94.

43. Minami Y, Kudo M, Hatanaka K, Kitai S, Inoue T, et al. Radiofrequency ablation guided by contrast harmonic sonography using perfluorocarbon microbubbles (Sonazoid) for hepatic malignancies: an initial experience. Liver Int 2010;30:759-64.

44. Masuzaki R, Shiina S, Tateishi R, Yoshida H, Goto E, et al. Utility of contrast-enhanced ultrasonography with Sonazoid in radiofrequency ablation for hepatocellular carcinoma. J Gastroenterol Hepatol 2011;26:759-64.

45. Dohmen T, Kataoka E, Yamada I, Miura K, Ohshima S, et al. Efficacy of contrast-enhanced ultrasonography in radiofrequency ablation for hepatocellular carcinoma. Intern Med 2012;51:1-7.

46. Minami T, Minami Y, Chishina H, Arizumi T, Takita M, et al. Combination guidance of contrast-enhanced US and fusion imaging in radiofrequency ablation for hepatocellular carcinoma with poor conspicuity on contrast-enhanced US/fusion imaging. Oncology 2014;87:55-62.

47. Huang DY, Yusuf GT, Daneshi M, Ramnarine R, Deganello A, et al. Contrast-enhanced ultrasound (CEUS) in abdominal intervention. Abdom Radiol (NY) 2018;43:960-76.

48. Kono Y, Lucidarme O, Choi SH, Rose SC, Hassanein TI, et al. Contrast-enhanced ultrasound as a predictor of treatment efficacy within 2 weeks after transarterial chemoembolization of hepatocellular carcinoma. J Vasc Interv Radiol 2007;18:57-65.

49. Xia Y, Kudo M, Minami Y, Hatanaka K, Ueshima K, et al. Response evaluation of transcatheter arterial chemoembolization in hepatocellular carcinomas: the usefulness of sonazoid-enhanced harmonic sonography. Oncology 2008;75:99-105.

50. Zhou P, Kudo M, Minami Y, Chung H, Inoue T, et al. What is the best time to evaluate treatment response after radiofrequency ablation of hepatocellular carcinoma using contrast-enhanced sonography? Oncology 2007;72:92-7.

51. Shiozawa K, Watanabe M, Kikuchi Y, Kudo T, Maruyama K, et al. Evaluation of sorafenib for hepatocellular carcinoma by contrastenhanced ultrasonography: a pilot study. World J Gastroenterol 2012 28;18:5753-8.

52. Sugimoto K, Moriyasu F, Saito K, Rognin N, Kamiyama N, et al. Hepatocellular carcinoma treated with sorafenib: early detection of treatment response and major adverse events by contrast-enhanced US. Liver Int 2013;33:605-15. 